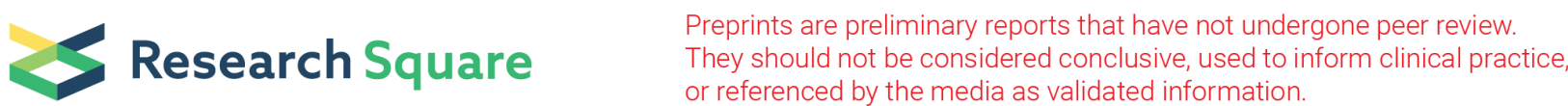

\section{Fatal Cross-Border Plague in Zombo District, Uganda, March 2019}

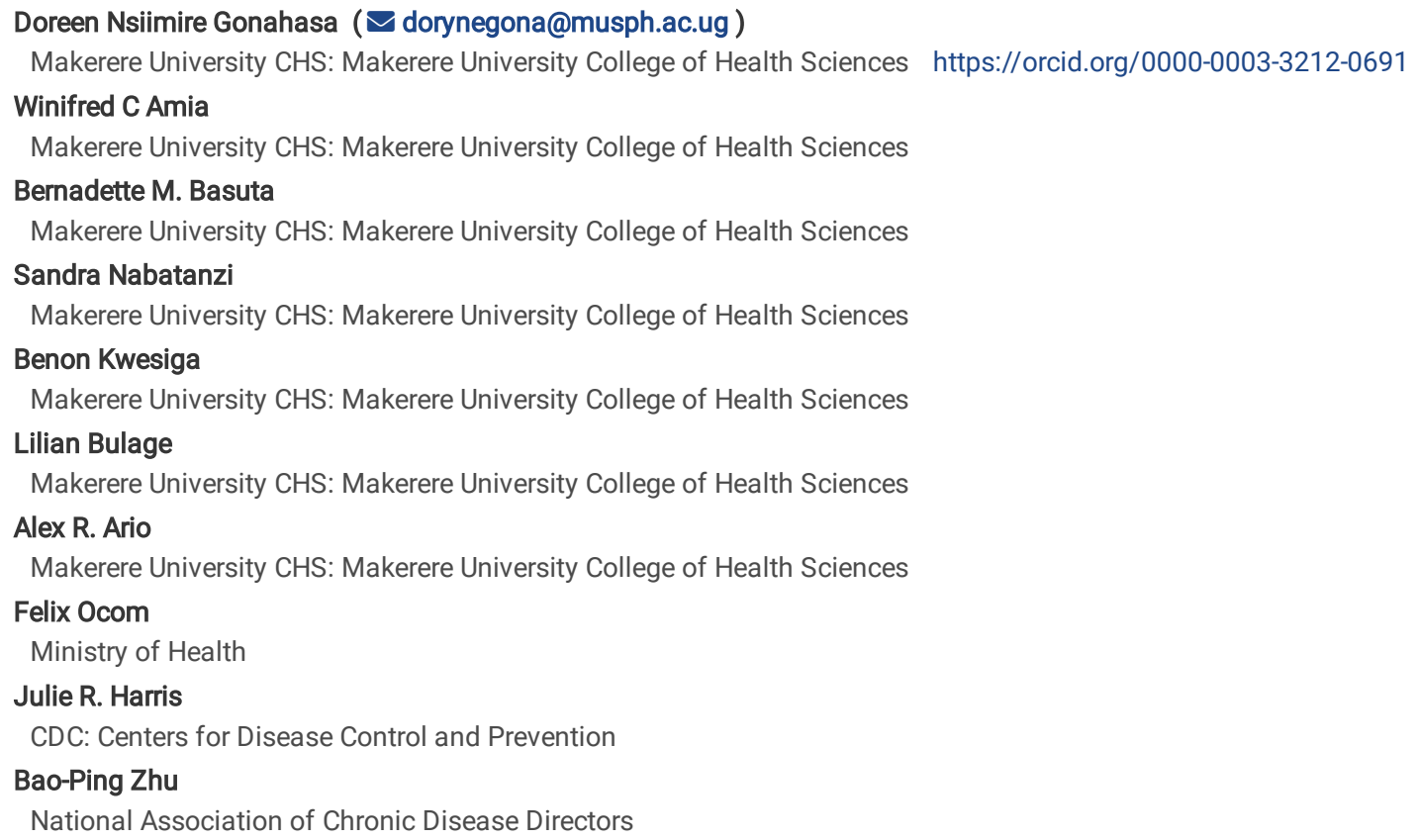

\section{Short Report}

Keywords: RDT, DRC, Case-patient

Posted Date: October 19th, 2020

DOI: https://doi.org/10.21203/rs.3.rs-91908/v1

License: (9) (7) This work is licensed under a Creative Commons Attribution 4.0 International License. Read Full License 


\section{Abstract}

Background: Plague, which is caused by Yersinia pestis, is a priority zoonotic disease targeted for elimination in Uganda. Untreated bubonic plague, resulting from a flea bite, can develop into pneumonic plague, or pneumonic plague may be transmitted in respiratory droplets from person to person. On 6 March 2019 , the Uganda Ministry of Health was notified of a patient in Zombo District with clinical presentation similar to pneumonic plague, and a positive plague rapid diagnostic test (RDT). The patient had family links to the Democratic Republic of Congo (DRC). We determined the scope of the outbreak, determined the mode of transmission, and recommended evidence-based control and prevention measures.

Methods: A suspected pneumonic plague case was $\geq 2$ of: cough (bloody or wet), chest pain, difficulty in breathing, or fever in a resident of Zombo District during February 1-March 31, 2019. A confirmed case was a suspected case testing positive for Yersinia pestis by rapid diagnostic test, culture or serology. We actively searched for case-patients, traced contacts and took samples as appropriate. We performed descriptive epidemiology of the outbreak.

Results: We identified one suspected and one confirmed pneumonic plague case. On February 26, 2019, a 4-year-old boy was buried in DRC near the Uganda border after reportedly succumbing to a disease consistent with bubonic plague. Case-patient A (35-year-old mother to the boy), fell ill with suspected pneumonic plague while attending to him. She was referred to a health facility in Uganda on February 28 but died on arrival. On March 4, Case-patient B (23year-old sister to Case-patient A), who attended to Case-patient A, presented with pneumonic plague symptoms to the same Uganda facility and tested plaguepositive by RDT, culture, and serological tests. Contacts $(n=114)$ were traced and given antibiotics as prophylaxis; no new cases were reported.

Conclusion: This fatal plague outbreak started as bubonic and later manifested as pneumonic. There was cross-border spread from DRC to Uganda with no cross-border efforts at prevention and control. Person-to-person transmission appears to have occurred. The quick and effective response likely minimized spread.

\section{Background}

Plague is a zoonotic disease caused by Yersinia pestis and found in wild rodents and their fleas [1]. It may present as bubonic, pneumonic, or septicemic plague [2]. Bubonic plague, which results when an infected flea bites a human, manifests as swollen nymph nodes called buboes. These may be inflamed and painful and may turn into open sores filled with pus in advanced stages. Pneumonic plague is the most virulent form and may result from progression of untreated bubonic plague or be transmitted by droplets from an infected person[2]. Septicemic plague manifests by darkening of parts of the body caused by infestation of the blood vessels by the bacteria [3]. The incubation period of plague ranges between 1-8 days, depending on the mechanism of infection and subsequent presentation [4]. Untreated plague has a fatality rate of $50-60 \%$ [5], but may be as high as $100 \%$ in septicemic plague cases [6].

At least three plague pandemics have been documented, each of which have left reservoirs in affected areas, including Europe, Asia, and Africa [2]. Although sporadic cases have occurred globally in recent years [7], improved sanitation has limited its spread [8]. Advancements in diagnostics and access to appropriate antibiotic therapy have also substantially reduced case-fatality rates [9]. Despite the decrease in human plague cases, plague bacteria continue to circulate in rodent hosts and their fleas in plague-endemic areas, including Madagascar [10], Democratic Republic of the Congo (DRC) [11], and northwestern Uganda [12].

Plague is considered a priority zoonotic disease in Uganda and is targeted for elimination [13]. Plague has been documented for decades in West Nile region, in north-western Uganda, as well as the neighbouring Ituri Province in DRC [11]. West Nile Region is a densely-populated region with a considerable refugee population [14]. It is located near the borders of DRC and South Sudan and predominantly has a subsistence agricultural economy. Over the years, there have been sporadic cases of plague in this region, with the overall incidence decreasing gradually since 2009 [3]. The decline in plague cases has likely been due to efforts by the Uganda Virus Research Institute to conduct plague surveillance and community sensitization on prevention methods in the region, including availing of RDT kits in the region [3].

On 6 March 2019, The Uganda Ministry of Health Public Health Emergency Operations Center (PHEOC) was notified of an adult female presenting with fever, haemoptysis, and difficulty breathing admitted at Warr Health Center III in Zombo District. The patient tested positive by plague by RDT. It was reported that her family members had travelled during the past week to DRC for burial of a person who had died with similar symptoms. The patient had returned to Uganda with her ailing sister, whom she nursed, and other family members. We investigated to determine the scope of the outbreak and the mode of transmission, and recommended evidence-based control and prevention measures.

\section{Methods}

\section{Outbreak area}

The outbreak occurred in Warr Sub-county, Zombo District. Zombo District is bordered by the DRC, about 20 km from Mahagi, Ituri Province where there had been an ongoing plague outbreak (Fig. 1). The district is located in the West Nile region in northwestern Uganda, the current plague focus of Uganda.

\section{Case definition and finding}

We defined a suspected pneumonic plague case was $\geq 2$ of: cough (bloody or wet), chest pain, difficulty breathing, or fever in a resident of Zombo District during February 1-March 31, 2019. A confirmed case was a suspected case testing positive for Yersinia pestis by rapid diagnostic test, culture or serology.

We actively searched for cases within the communities through support by village health teams (VHTs). We also visited three health facilities (Warr HCIII, Kango HCll, and Holy Family Hospital, Nyapea) where we reviewed patient records to identify possible cases of pneumonic plague. 


\section{Contact tracing and follow up}

We traced both primary and secondary contacts of the suspected case-patient using the World Health Organization (WHO) Integrated Disease Surveillance and Response (IDSR) contact tracing form [13]. We defined primary contacts as persons who cared for other case-patients or stayed in the same room with them, while secondary contacts were those that stayed in the same household or facility where the case-patients were managed [15]. Contacts were followed for seven days to observe any symptom development.

\section{Laboratory Investigations}

Sputum and whole blood were taken from the single surviving case-patient. From these samples, a plague RDT at Warr Health Centre, culture, and PCR for plague at the CDC laboratory in Atlanta, USA were conducted. Multiple tests were used to help provide rapid results (RDT) and have confidence in results (culture).

\section{Ethical Considerations}

The Uganda Ministry of Health gave the directive and approval to investigate this outbreak. The Office of the Associate Director for Science at the CDC's Center for Global Health determined that this activity was not human subjects research and that its primary intent was disease control. Verbal informed consent in the local language was sought from respondents. They were informed that their participation was voluntary, and their refusal would not result in any negative consequences. Contacts who received prophylaxis were informed of its benefits and possible risks.

\section{Results}

\section{Chronology of events and case-patients' description}

On February 26, 2019, a 4-year-old Congolese boy was buried in Mahagi Parish of the Ituri Province of DRC, near the Ugandan border where his family lived. On February 28, 2019, Case-patient A, the 35-year-old mother of the boy, also a resident of DRC, was brought to Warr Health Centre III in Zombo District, Uganda, by Ugandan family members. She was febrile (temperature not recorded), coughing blood, and reporting difficulty breathing and chest pain. Her family members reported that she had been caring her ill son in DRC before he died. The family members, who had traveled to DRC to attend the burial of her son, noted that she was ill at the burial and brought her back to Uganda for treatment. Unfortunately, case-patient A died shortly after arrival at the hospital. Healthcare workers did not administer any diagnostic tests. The movement routes of the family from the DRC to Uganda are shown in Fig. 1.

On 4 March, case-patient B, the 23-year-old sister to case-patient A presented at Warr HCIII with headache, fever, chills, sore throat, chest pain, difficulty breathing, coughing blood, malaise, prostration, and anorexia. Case-patient B had cared for case-patient A during her illness. An RDT conducted on sputum sample from case-patient B was positive for plague. She was immediately isolated and managed with oral ciprofloxacin until recovery. Further confirmatory tests by culture and PCR were also positive for Yersinia pestis.

\section{Contact tracing and follow up findings}

We identified 114 contacts for both case-patients, including 44 primary contacts and 59 secondary contacts. Eleven (9.6\%) of the contacts were healthcare workers. Of the 114 contacts, $65 \%$ were female and median age was 40 years (Range: $1-75$ years). All contacts were given doxycycline (for primary contacts that came into direct contact with patient body fluids) or cotrimoxazole (for secondary contacts who were in close proximity with the patient). Contacts were followed for seven days and observed for signs and symptoms of plague. No new cases were identified. The nearby communities were sensitized on plague prevention, and healthcare workers at Warr Health Centre were provided with a continuing medical education course on plague.

\section{Discussion}

Our epidemiological investigation revealed a pneumonic plague outbreak in Uganda imported from the DRC. The outbreak represented human-to-human transmission in a single family. Timely response was facilitated by health workers with a high index of suspicion, availability of rapid test kits, and a functional surveillance system that enabled rapid identification and response. This rapid response likely minimized spread.

The Ituri Province of the DRC is endemic for plague, and the province reported additional cases of plague in late 2019 [16] as well as during June 2020 [17]. Northwestern Uganda also experiences sporadic plague cases [3]. Both of these areas lie at similar altitudes (>1300 m), with lower temperatures and higher rainfall than the surrounding lowlands [12]. Such areas, along with Rift Valley escarpments, have been found to be at elevated risk for plague [9]. Hotspots of pneumonic plague in Madagascar have also been identified in ecologically similar regions, in the northeastern central highlands, where the median elevation is $1200-1300 \mathrm{~m} \mathrm{[18].}$

Despite sporadic cases of plague in Uganda, the incidence has gradually been decreasing [3]. This may be in part attributed to an enhanced surveillance program begun in 2003 by the Uganda Virus Research Institute (UVRI). The UVRI Plague Program has been actively involved in surveillance of plague in the West Nile Region with the aim of understanding the plague ecology and epidemiology, developing appropriate diagnostics, patient treatment and care guidelines, and effective public health interventions. This program has also included sensitization of communities about plague, which may have led to greater community awareness and prevention measures [3].

Case-patient A likely died as a result of delayed care and treatment. She was brought from the DRC to the health center in Uganda after her family found she had already been ailing. Pneumonic plague in adults is usually attributed to delayed diagnosis of bubonic plague and subsequent disease progression; the average time from full onset of pneumonic plague to death is 1.9 days [18]. Because of the rapid progression to death, it is essential to obtain laboratory 
diagnosis and treatment as quickly as possible [19]. However, the nonspecific presentation of pneumonic plague can complicate diagnosis [18], and having access to field-appropriate diagnostics in low-resource settings is critical to preventing plague deaths. Plague RDT is an field-appropriate tool that has led to early detection of outbreaks and rapid implementation of control measures in multiple countries in Africa [3].

Historical documentation of plague in Africa dates back to the early 1900s [20], though it likely occurred well before then. These outbreaks have ranged in size from dozens to thousands of cases [21]. A 2017 outbreak in Madagascar ultimately affected over 2,000 persons, with 202 deaths (case-fatality rate $=8.6 \%$ ) [19]; this outbreak spread quickly in part due to a weak surveillance system [23]. In contrast, the outbreak in Zombo District was likely contained by early detection and response in the form of isolation and treatment of the suspected case, contact tracing, and prophylaxis for contacts. Having access to tools for early detection and treatment, as well as ensuring adequate training of health workers, are critical to addressing plague outbreaks and reducing overall plague deaths [18]. The Ministry of Health should focus efforts on sustained surveillance for early detection and timely response to outbreaks of this nature.

\section{Limitations}

While the outbreak of plague was imported from the DRC, we were unable to travel there to investigate the root cause of this outbreak, due to lack of clear cross-border collaboration mechanisms in emergency and outbreak management at the time. Fortunately, collaborative efforts have improved recently, in large part due to relationships fostered by the Ebola Virus Disease outbreak that has been ongoing for more than a year.

\section{Conclusion And Recommendations}

This outbreak of plague affected one family in Uganda and was imported from the DRC. Timely response in form of prompt identification, isolation, and treatment of case-persons, tracing of contacts, and antibiotic prophylaxis for the contacts were effective in containing this outbreak.

We recommended strengthening of cross-border collaborations and surveillance led by the Ministries of Health in both Uganda and DRC to ensure early detection and containment of plague outbreaks. This recommendation is strengthened by evidence of gains realised during the Ebola Virus Disease outbreak in DRC during 2018-2019. Furthermore, major health facilities in the risk area should be provided with more plague RDT kits.

\section{List Of Abbreviations}

CDC: Centers for Disease Control and Prevention; DRC: Democratic Republic of Congo; HC; Health Centre; IDSR: Integrated Disease Surveillance and Response; PHEOC: Public Health Emergency Operations Centre; RDT: Rapid Diagnostic Test; WHO: World Health Organization

\section{Declarations}

\section{Ethical approval and Consent to participate}

This investigation was in response to a public health emergency and was therefore determined to be non-research. Uganda Ministry of Health gave the directive and approval to investigate this outbreak. The Office of the Associate Director for Science at the US CDC's Center for Global Health determined that this activity was not human subjects research, and that its primary intent was public health practice or a disease control activity. Verbal informed consent in the local language was sought from respondents. They were informed that their participation was voluntary, and their refusal would not result in any negative consequence.

\section{Consent for publication}

There are no images or personalised data used in this manuscript,

\section{Availability of supporting data and materials}

The datasets upon which our findings are based belong to the Uganda Public Health Fellowship Program, Ministry of Health. For confidentiality reasons the datasets are not publicly available. However, the data sets can be availed upon reasonable request from the corresponding author and with permission from the Uganda Public Health Fellowship Program or Ministry of Health.

\section{Competing interests}

The authors declare that they had not competing interests.

\section{Funding and Disclaimer}

This project was supported by the President's Emergency Plan for AIDS Relief (PEPFAR) through the US Centers for Disease Control and Prevention Cooperative Agreement number GH001353-01 through Makerere University School of Public Health to the Uganda Public Health Fellowship Program, Ministry of Health. Its contents are solely the responsibility of the authors and do not necessarily represent the official views of the US Centers for Disease Control and Prevention, the Department of Health and Human Services, Makerere University School of Public Health, or the Uganda Ministry of Health. The staff of the funding body provided technical guidance in the design of the study, ethical clearance and collection, analysis, and interpretation of data, and in writing the manuscript.

\section{Authors' contribution}


DNG took lead in execution of the investigation. She wrote the drafts of the manuscript and revised the paper for substantial intellectual content. WA, BMB and SN participated in the conceptual design and development of the investigation and were involved in field data collection as well as data analysis. They also reviewed the paper for substantial intellectual content. BK, LB, ARA, FO, JH, and BPZ participated in designing the investigation, supervision of field data collection, data analysis, and reviewed the draft manuscript for substantial intellectual content. All authors have read and approved the final manuscript.

\section{Acknowledgements}

We acknowledge the Zombo District Local Government for their technical support and effort during the response to this outbreak. We appreciate the Uganda Virus Research Institute for specimen testing to confirm the etiology of this outbreak.

\section{Authors' information}

*Doreen N. Gonahasa: dorynegona@musph.ac.ug, Winifred C. Amia: amiawiny@gmail.com, Bernadette M. Basuta: bagheni@musph.ac.ug , Sandra Nabatanzi: sandranabatanzi@musph.ac.ug, Benon Kwesiga: benon.kwesiga@musph.ac.ug, Lilian Bulage: Ibulage@musph.ac.ug , Alex R. Ario: riolexus@musph.ac.ug, Felix Ocom: felixocom@gmail.com,Julie R. Harris: ggt5@cdc.gov,Bao-Ping Zhu: bpzhu@yahoo.com

\section{References}

1. CDC. Plague home | CDC [Internet]. Centers for Disease Control and Prevention. 2019 [cited 2020 May 30]. Available from: /plague/index.html

2. WHO Plague Fact sheet. [cited 2020 May 30]. Available from: https://www.who.int/news-room/fact-sheets/detail/plague

3. Forrester JD, Apangu T, Griffith K, Acayo S, Yockey B, Kaggwa J, et al. Patterns of Human Plague in Uganda, 2008-2016 - Volume 23, Number 9September 2017 - Emerging Infectious Diseases journal - CDC. [cited 2019 Oct 18]; Available from: https://wwwnc.cdc.gov/eid/article/23/9/170789_article

4. Control of Communicable Diseases Manual [Internet]. [cited 2019 Mar 18]. Available from: https://www.apha.org/ccdm

5. WHO Report on Global Surveillance of Epidemic-prone Infectious Diseases [cited 2019 Nov 20]. Available from: http://www.bioline.org.br/pdf?nd12051

6. Septicemic Plague - an overview I ScienceDirect Topics [Internet]. [cited 2020 May 30]. Available from:

https://www.sciencedirect.com/topics/pharmacology-toxicology-and-pharmaceutical-science/septicemic-plague

7. RT Madagascar20_09_2018.pdf [Internet]. [cited 2019 Sep 9]. Available from: https://extranet.who.int/sph/sites/default/files/documentlibrary/document/RT\%20Madagascar20_09_2018

8. Strengthening Foodborne Diseases Surveillance in the Who African Region: An Essential Need for Disease Control and Food Safety Assurance. 2012;12(4):18

9. Eisen RJ, MacMillan K, Atiku LA, Mpanga JT, Zielinski-Gutierrez E, Graham CB, et al. Identification of Risk Factors for Plague in the West Nile Region of Uganda. Am J Trop Med Hyg. 2014 Jun 4;90(6):1047-58.

10. Drancourt M, Raoult D. Investigation of Pneumonic Plague, Madagascar. Emerg Infect Dis. 2018 Jan;24(1):183.

11. Abedi AA, Shako J-C, Gaudart J, Sudre B, llunga BK, Shamamba SKB, et al. Ecologic Features of Plague Outbreak Areas, Democratic Republic of the Congo, 2004-2014 - Volume 24, Number 2-February 2018 - Emerging Infectious Diseases journal - CDC. [cited 2019 Oct 17]; Available from: https://wwwnc.cdc.gov/eid/article/24/2/16-0122_article

12. Al JDF et. Patterns of Human Plague in Uganda, 2008-2016 - Volume 23, Number 9-September 2017 - Emerging Infectious Diseases journal - CDC. [cited 2019 Mar 18]; Available from: https://wwwnc.cdc.gov/eid/article/23/9/17-0789_article

13. National Technical Guidelines for Integrated Disease Surveillance and Response

14. Uganda Country RRP 2019-20 (January 2019).pdf [Internet]. [cited 2020 May 30]. Available from: http://reporting.unhcr.org/sites/default/files

15. WHO_EVD_Guidance_Contact_15.1_eng.pdf [Internet]. [cited 2020 Mar 18]. Available from: https://apps.who.int/iris/bitstream/handle/10665/185258/WHO_EVD_Guidance_Contact_15.1_eng.pdf;jsessionid=F9AA97CDA5655EBBA1004C18C7583 sequence $=1$

16. Plague: A dozen additional cases reported in Ituri province, DRC [Internet]. Outbreak News Today. 2019 [cited 2020 May 30]. Available from: http://outbreaknewstoday.com/plague-a-dozen-additional-cases-reported-in-ituri-province-drc-12148/

17. Plague cluster reported in Ituri, Democratic Republic of Congo [Internet]. Outbreak News Today. 2020 [cited 2020 Jul 14]. Available from: http://outbreaknewstoday.com/plague-cluster-reported-in-ituri-democratic-republic-of-congo-45293/

18. Al VA et. Trends of Human Plague, Madagascar, 1998-2016 - Volume 25, Number 2-February 2019 - Emerging Infectious Diseases journal - CDC. [cited 2019 Mar 18]; Available from: https://wwwnc.cdc.gov/eid/article/25/2/17-1974_article

19. WHO | Revamp of the plague detection in Madagascar yields quick and sustainable wins [Internet]. WHO. [cited 2019 Apr 29]. Available from: http://www.who.int/csr/disease/plague/laboratory-detection-madagascar/en/

20. Davis DHS. A Survey of Wild Rodents in African Territories. :37.

21. Lotfy WM. Current perspectives on the spread of plague in Africa [Internet]. Vol. 6, Research and Reports in Tropical Medicine. Dove Press; 2015 [cited 2020 Jun 2]. p. 21-30. Available from: https://www.dovepress.com/current-perspectives-on-the-spread-of-plague-in-africa-peer-reviewed-fulltext-articleRRTM

22. WHO | Plague - Madagascar [Internet]. WHO. World Health Organization; [cited 2020 Jun 14]. Available from: http://www.who.int/csr/don/27-november2017-plague-madagascar/en/ 
23. October 18 DS, 2017. How the Plague Outbreak in Madagascar Got So Bad, So Fast [Internet]. livescience.com. [cited 2020 Jun 14]. Available from: https://www.livescience.com/60715-plague-outbreak-madagascar.html

\section{Figures}

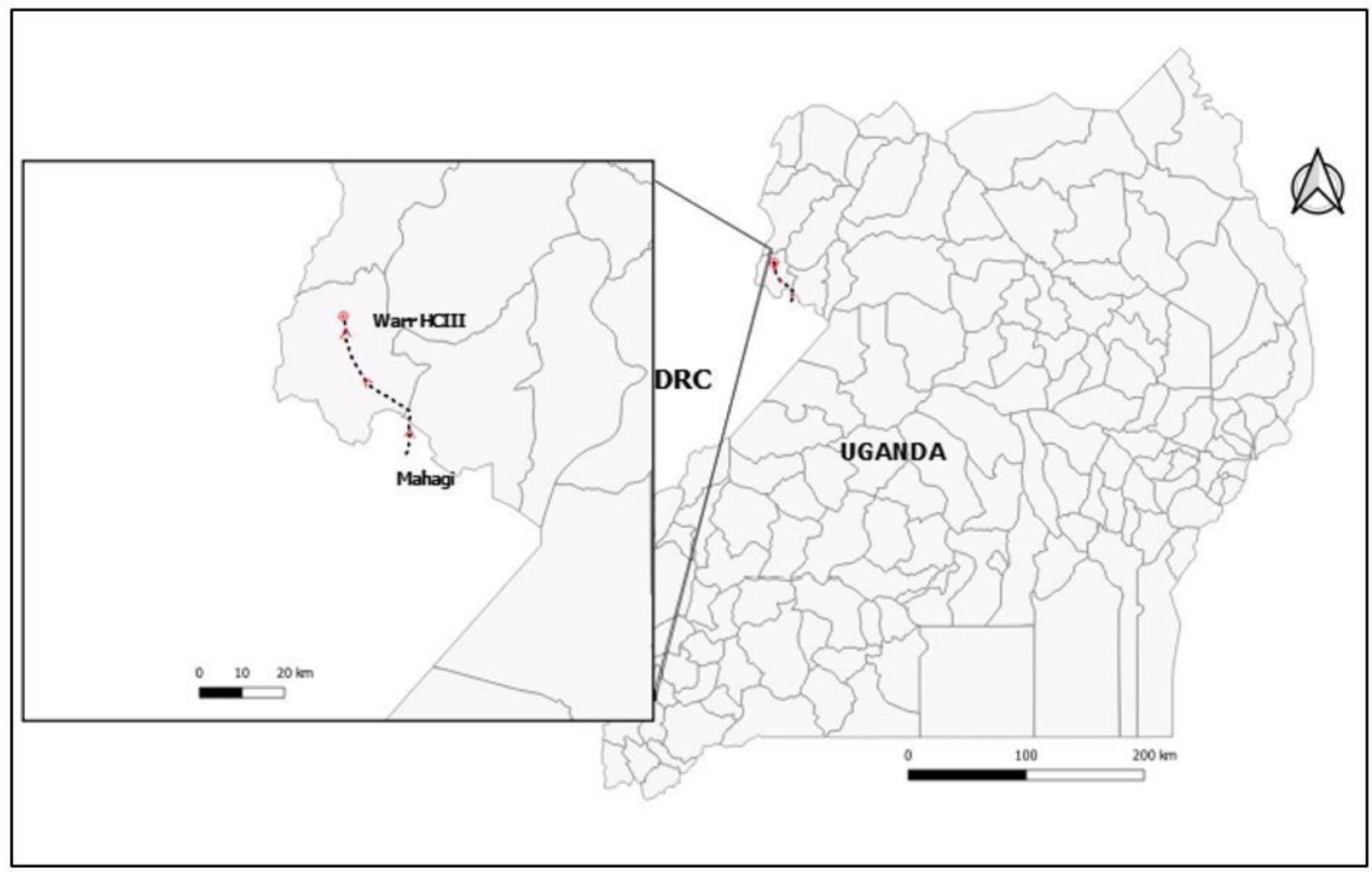

Figure 1

Map showing movement of case-patient A from Mahagi in DRC to Warr HCIII in Zombo, Uganda. Four plague cases were reported in Mahagi, Ituri Province, one of whom traveled to Warr HCll for treatment on 28th March 2019 Note: The designations employed and the presentation of the material on this map do not imply the expression of any opinion whatsoever on the part of Research Square concerning the legal status of any country, territory, city or area or of its authorities, or concerning the delimitation of its frontiers or boundaries. This map has been provided by the authors. 